\title{
Nonlinear Standing Waves in a Layer Excited by the Periodic Motion of its Boundary ${ }^{1}$
}

\author{
O. V. Rudenko*, C. M. Hedberg**, B. O. Enflo*** \\ *Moscow State University, Vorob'evy gory, Moscow, 119899 Russia \\ e-mail: rudenko@acs366b.phys.msu.su \\ **University of Karlskrona/Ronneby, 37179 Karlskrona, Sweden \\ ***Royal Institute of Technology, S-10044 Stockholm, Sweden
}

Received August 24, 2000

\begin{abstract}
A new analytical approach is developed for the description of standing waves caused by arbitrary periodic vibration of a boundary. The approach is based on the nonlinear evolution equation written for an auxiliary function. This equation offers the possibility to study not only the steady-state acoustic field, but also its evolution in time. One can take into account the dissipative properties of the medium and the difference between one of the resonant frequencies and the fundamental frequency of the driving motion of the wall. An exact nonsteady-state solution is derived corresponding to the sawtooth-like periodic vibration of the boundary. The maximal "amplitude" values of the particle velocity and the energy of a standing wave are calculated. The temporal profiles of standing waves at different points of the layer are presented. A new possibility of pumping a high acoustic energy into a resonator is indicated for the case of a special type of the wall motion having the form of an "inverse saw." Theoretically, such a vibration leads to an "explosive instability" and an unlimited growth of the standing wave. For a harmonic excitation, the exact non-steady-state solution is derived as well. The standing wave profiles are described by Mathieu functions, and the energy characteristics by their eigenvalues. () 2001 MAIK "Nauka/Interperiodica".
\end{abstract}

\section{INTRODUCTION}

Standing waves are of great interest for nonlinear science and its applications $[1,2]$. Theoretical and experimental studies have been made during the last few decades (see, for example, [3-6]). Scientific interest is stimulated by the possibility to accumulate considerable energy in a high- $Q$ resonant system and to form conditions for the observation of well-defined nonlinear phenomena even with a weak driving source. In such a system, the linear losses are weak and the nonlinear absorption plays the role of a limiting factor.

The $Q$-factor shows how many times the amplitude of the stationary wave is higher than the amplitude of vibration of the boundary. Because of nonlinear absorption, the $Q$-factor of a given resonator depends on this amplitude. As a stationary regime is approached, the amplitude of a standing wave increases and nonlinear effects become more and more important. The wave profiles, the $Q$-factor, and all other characteristics of the wave field now depend on time.

However, the non-steady-state nonlinear vibrations in cavities have been studied much less than the stationary ones. The main objective of this work is to develop an analysis of the transient processes in a one-dimensional layer and to derive the corresponding analytical results.

\footnotetext{
${ }^{1}$ This article was submitted by the authors in English.
}

\section{SIMPLIFIED EVOLUTION EQUATION}

It is evident that in a linear one-dimensional system, a standing wave can be composed of two plane waves propagating in opposite directions. This idea has been generalized for a nonlinear standing wave between rigid immovable walls [7] where the vibration in the layer is described as the sum of two Riemann or Burgers travelling waves. These waves can be distorted significantly by a nonlinear self-action, which results in the formation of a sawtooth-like profile in place of the initial harmonic one with no contribution made by the cross-interaction of the initial harmonic waves. In other words, each wave is distorted by itself during the propagation, but no energy exchange occurs between them. A similar approach has been used [8] to describe the nonlinear field between two parallel rigid planes; in this case, the nonlinear Brillouin modes are formed by two strongly distorted waves intersecting at equal angles the axis of a waveguide. Despite the existing works, this idea is not well proven and needs to be considered in more detail.

The explanation given below is valid when any nonlinear equation describing nondispersive waves in a quadratic nonlinear medium is used. Here, to be specific, a model one-dimensional equation is analysed [9]:

$$
\frac{\partial^{2} p}{\partial x^{2}}-\frac{1}{c^{2}} \frac{\partial^{2} p}{\partial t^{2}}=-\frac{\varepsilon}{c^{4} \rho} \frac{\partial^{2} p^{2}}{\partial t^{2}} .
$$


Here, $p$ is the acoustic pressure, $c$ and $\rho$ are the sound velocity and the density of the medium, and $\varepsilon$ is the nonlinearity. Equation (1) can govern the Riemann waves propagating in opposite directions, as well as the interaction between them. The solution is sought for by a successive approximation method:

$$
p=p^{(1)}+p^{(2)}+\ldots
$$

Let the first-approximation solution be a sum of two waves:

$$
\begin{gathered}
p^{(1)}=B_{1} \cos \left(\omega_{1} t-k_{1} x+\varphi_{1}\right) \\
+B_{2} \cos \left(\omega_{2} t+k_{2} x+\varphi_{2}\right), \quad k_{1,2}=\omega_{1,2} / c .
\end{gathered}
$$

The second approximation is derived from the inhomogeneous linear equation

$$
\begin{gathered}
\frac{\partial^{2} p^{(2)}}{\partial x^{2}}-\frac{1}{c^{2}} \frac{\partial^{2} p^{(2)}}{\partial t^{2}} \\
=F\left(2 \omega_{1}\right)+F\left(2 \omega_{2}\right)+F\left(\omega_{1}+\omega_{2}\right)+F\left(\omega_{1}-\omega_{2}\right),
\end{gathered}
$$

the right-hand side of which is calculated on the base of the first-approximation solution (3)

$$
\begin{gathered}
F\left(2 \omega_{1,2}\right)=\frac{2 \varepsilon}{c^{4} \rho} \omega_{1,2}^{2} B_{1,2}^{2} \\
\times \cos \left[2\left(\omega_{1,2} t \mp k_{1,2} x+\varphi_{1,2}\right)\right], \\
F\left(\omega_{1} \pm \omega_{2}\right)=\frac{\varepsilon}{c^{4} \rho}\left(\omega_{1} \pm \omega_{2}\right)^{2} B_{1} B_{2} \\
\times \cos \left[\left(\omega_{1} \pm \omega_{2}\right) t-\left(k_{1} \mp k_{2}\right) x+\left(\varphi_{1} \pm \varphi_{2}\right)\right] .
\end{gathered}
$$

In the context of the approximate method (2), the four terms on the right-hand side of Eq. (4) can be considered as "external forces" exciting the second-approximation forced waves at the frequencies of second harmonics $2 \omega_{1}$ and $2 \omega_{2}$, as well as at the sum $\left(\omega_{1}+\omega_{2}\right)$ and difference $\left(\omega_{1}-\omega_{2}\right)$ frequencies.

It is important that the excitation of secondary waves can have a resonant character or a nonresonant one. The first two "forces" given by Eq. (5), $F\left(2 \omega_{1}\right)$ and $F\left(2 \omega_{2}\right)$, lead to the resonant excitation. The corresponding forced waves

$$
\begin{gathered}
p_{1,2}^{(2)} \\
=-\frac{\varepsilon}{2 c^{2} \rho} B_{1,2}^{2}\left(\omega_{1,2} t\right) \sin \left[2\left(\omega_{1,2} t \mp k_{1,2} x+\varphi_{1,2}\right)\right]
\end{gathered}
$$

increase in time. The amplitudes grow linearly with time $t$ like the amplitude of forced vibration of a pendulum at the coincidence of the eigen- and driving frequencies.

In contrast to systems with one degree of freedom, the resonance in spatially distributed systems occurs at the coincidence of the velocities of motion of both driving force and exciting wave [9].
Unlike solution (6) that increases in amplitude with $t$, the partial solution of Eq. (4) corresponding to other two forced waves excited by the forces $F\left(\omega_{1} \pm \omega_{2}\right)$ from Eqs. (5)

$$
\begin{gathered}
p_{3,4}^{(2)}=\frac{\varepsilon}{c^{4} \rho} \frac{\left(\omega_{1} \pm \omega_{2}\right)^{2}}{4 k_{1} k_{2}} B_{1} B_{2} \\
\times \cos \left[\left(\omega_{1} \pm \omega_{2}\right) t-\left(k_{1} \mp k_{2}\right) x+\left(\varphi_{1} \pm \varphi_{2}\right)\right],
\end{gathered}
$$

have amplitudes independent of $t$.

The comparison between the resonant (6) and nonresonant (7) solutions shows that after several periods of vibration, $\left(\omega_{1,2} t\right) \gg 1$, the waves described by Eq. (7) become much weaker than the resonant waves (6) and cannot participate significantly in the nonlinear energy exchange. Consequently, each of the two waves propagating in opposite directions generates its higher harmonics (6), but the cross-interaction processes (7) can be neglected if the waves are periodic in time. This conclusion is easily seen to be valid for periodic waves intersecting at any sufficiently large angles depending on the acoustic Mach number [8].

Let us now start with the derivation of the simplified evolution equation by taking into account, as the first step, the nonlinear processes only. We consider a plane layer. Its left boundary can vibrate according to the law

$$
u(x=0, t)=A f(\omega t),
$$

where $A$ is a characteristic amplitude, $u$ is velocity, and $f$ is a periodic function of its argument with the period $2 \pi$. The right boundary is immovable:

$$
u(x=L, t)=0 .
$$

In order to apply the boundary conditions (8) and (9) to the wave equation (1), we write this equation as a Riemann one for the velocity. This can be done at the same time for right- and leftgoing waves by using in Eq. (1) the relation $p= \pm \rho c u$, where the plus sign is taken for the rightgoing and the minus sign for the leftgoing waves, and making the rescaling $x \longrightarrow \mu x$, where $\mu$ is a small parameter. Neglecting the terms $O\left(\mu^{2}\right)$, after the restitution $\mu x \longrightarrow x$, we obtain the nonlinear equation [10] for Riemann waves

$$
\frac{\partial u}{\partial x} \pm \frac{1}{c} \frac{\partial u}{\partial t}-\frac{\varepsilon}{c^{2}} u \frac{\partial u}{\partial t}=0 .
$$

The upper (lower) sign in Eq. (10) corresponds to the wave propagating in the positive (negative) direction of the $x$ axis. The solution to Eq. (10) exists in the implicit form. The sum of two solutions can be written as

$$
\begin{gathered}
u=F\left[\omega t-\frac{\omega}{c}(x-L)+\frac{\varepsilon}{c^{2}} \omega(x-L) F\right] \\
-F\left[\omega t+\frac{\omega}{c}(x-L)-\frac{\varepsilon}{c^{2}} \omega(x-L) F\right],
\end{gathered}
$$


where $F$ is an auxiliary function describing the profile of a nonlinear traveling wave. One can see, that solution (11) satisfies the boundary condition (9). Condition (8) applied to Eq. (11) gives the functional equation

$$
\begin{gathered}
A f(\omega t)=F\left[\omega t+k L-\frac{\varepsilon}{c} k L F\right] \\
-F\left[\omega t-k L+\frac{\varepsilon}{c} k L F\right] .
\end{gathered}
$$

Here, the function $f(\omega t)$ is known, and the function $F(\omega t)$ must be calculated.

The functional equations of implicit form are complicated and cannot be solved analytically. In the simplest case, if we set the nonlinearity $\varepsilon=0$ and the function $f=\sin (\omega t)$ in Eq. (12), the functional equation

$$
F[\omega t+k L]-F[\omega t-k L]=A \sin (\omega t)
$$

can be solved easily. Its solution

$$
\begin{gathered}
F=-\frac{A \cos (\omega t)}{2 \sin (k L)} \\
+\sum_{n=0}^{\infty}\left[A_{n} \cos \left(n \omega_{0} t\right)+B_{n} \sin \left(n \omega_{0} t\right)\right]
\end{gathered}
$$

consists of the partial solution of the inhomogeneous equation (13) and the general solution of the corresponding homogeneous equation.

Here,

$$
\omega_{0}=\pi \frac{c}{L}
$$

where

$$
L=\frac{\lambda_{0}}{2}
$$

is the fundamental resonance frequency. If the driving frequency approaches a resonance one, $\omega \longrightarrow n \omega_{0}$, the non-steady-state process comes into play. To show this, let us set in solution (14):

$$
A_{m}=\frac{A}{2 \sin (k L)}, \quad B_{n}=0, \quad A_{n}=0 \quad(n \neq m) .
$$

Solution (14) with Eq. (15) has an uncertainty of the $\left(\frac{0}{0}\right)$-type. Solving this uncertainty, we obtain

$$
\begin{aligned}
F & =\frac{A}{2} \lim _{\omega \rightarrow n \omega_{0}} \frac{\cos \left(n \omega_{0} t\right)-\cos (\omega t)}{\sin \left(\frac{\omega L}{c}\right)} \\
& =\frac{A}{2 \pi}(-1)^{n}\left(\omega_{0} t\right) \sin \left(n \omega_{0} t\right) .
\end{aligned}
$$

Solution (16) describes the resonant vibration with an increasing amplitude. This simple example illustrates a surprising, at first sight, fact that the functional equations like Eqs. (12) and (13) can describe not only the steady-state vibration, but also different transient processes.

Let us now pass to the nonlinear functional equation (12). It can be reduced to the simplified evolution equation, if the length of the resonator is small in comparison with the nonlinear length, and the frequency of vibration of the left boundary differs weakly from the resonant frequency

$$
\begin{gathered}
L \ll \frac{c^{2}}{\varepsilon \omega F_{\max }}, \quad k L=\pi+\Delta, \\
\Delta=\pi \frac{\omega-\omega_{0}}{\omega_{0}} \ll 1 .
\end{gathered}
$$

Here, $F_{\max }$ is the maximal value of the function $F$, and $\Delta$ is a discrepancy. The second condition (17) corresponds to the fundamental resonance $(n=1)$, for definiteness. With such restrictions, the right-hand side of Eq. (12) can be expanded in a series:

$$
\begin{gathered}
F=\left[\omega t+\pi+\Delta-\pi \frac{\varepsilon}{c} F\right]-F\left[\omega t-\pi-\Delta+\pi \frac{\varepsilon}{c} F\right] \\
\approx[F(\omega t+\pi)-F(\omega t-\pi)] \\
+\left(\Delta-\pi \frac{\varepsilon}{c} F\right)\left[F^{\prime}(\omega t+\pi)+F^{\prime}(\omega t-\pi)\right] .
\end{gathered}
$$

It is evident that $F$ is a quasi-periodic function whose parameters slowly vary in time. Therefore,

$$
F(\omega t+\pi)-F(\omega t-\pi) \approx 2 \pi \mu \frac{\partial F}{\partial(\mu \omega t)},
$$

where $\mu \ll 1$ is a small parameter the physical meaning of which will be clear later. Equation (12) will have the form

$$
\frac{A}{2} f(\omega t)=\mu \frac{\partial F}{\partial\left(\frac{\mu}{\pi} \omega t\right)}+\left(\Delta-\pi \frac{\varepsilon}{c} F\right) \frac{\partial F(\omega t+\pi)}{\partial(\omega t)} .
$$

Introducing new dimensionless variables and constants

$$
\xi=\omega t+\pi, \quad U=\frac{F}{c}, \quad M=\frac{A}{c}, \quad T=\frac{\omega t}{\pi},
$$

one can rewrite Eq. (18) as

$$
\frac{\partial U}{\partial T}+\Delta \frac{\partial U}{\partial \xi}-\pi \varepsilon U \frac{\partial U}{\partial \xi}=\frac{M}{2} f(\xi-\pi) .
$$

It is now evident that the small parameter $\mu$ can play the role of any small number: $\Delta, M$, or $U \sim M$.

In recent works $[11,12]$, an equation similar to Eq. (19) was derived with allowance made for the dis- 


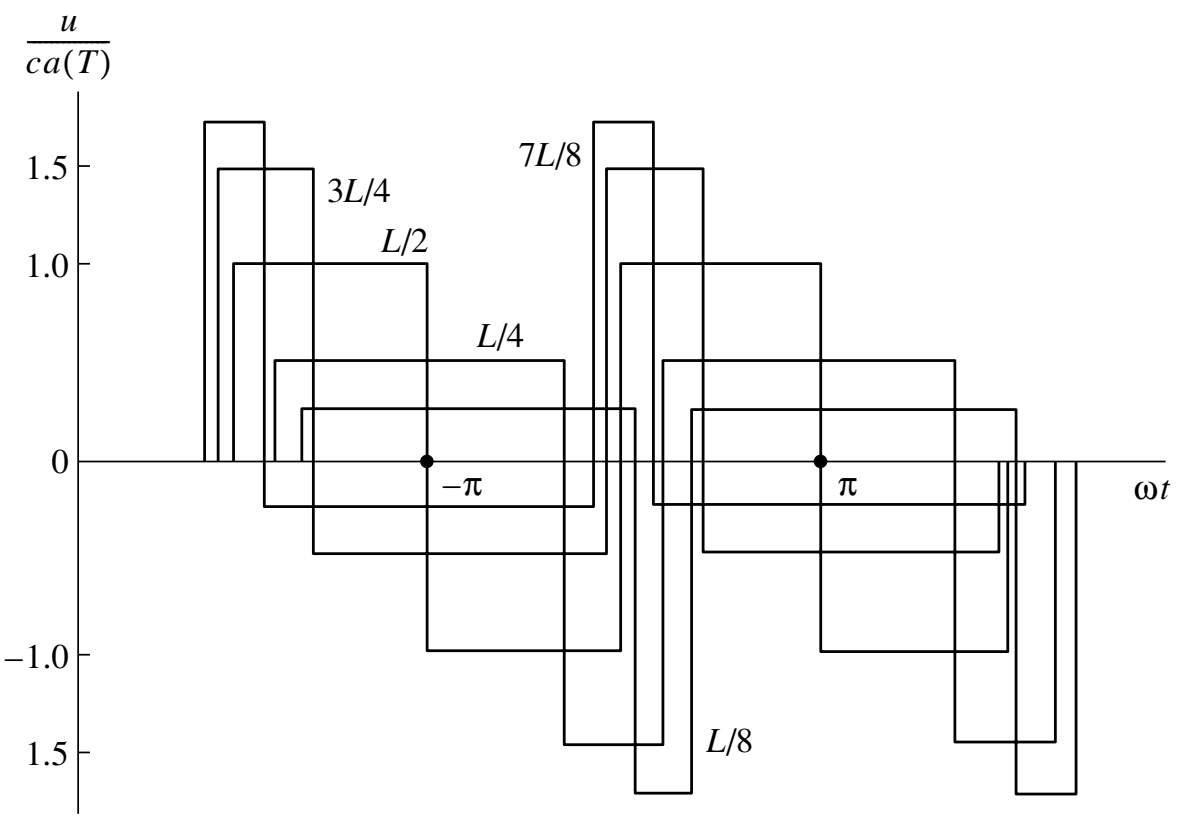

Fig. 1.

sipation and the finite displacement of the vibrating boundary:

$$
\frac{\partial U}{\partial T}-D \frac{\partial^{2} U}{\partial \xi^{2}}-M \varphi(\xi) \frac{\partial U}{\partial \xi}+\Delta \frac{\partial U}{\partial \xi}=\frac{M}{2} \varphi^{\prime}(\xi),
$$

where $\varphi^{\prime}(\xi)$ is a periodic function and

$$
D=\frac{b \omega^{2}}{2 c^{3} \rho} L \ll 1
$$

is the dimensionless number determining the weak absorption of a wave passing through the length $L$ of the resonator. However, the volume nonlinearity was not considered in $[11,12]$.

The main objective of this study is the simultaneous consideration of the weak effects of dissipation and nonlinearity. Because all the phenomena leading to a progressive distortion of the wave are supposed to be weak, the corresponding terms in the evolution equation must be additive $[9,10]$. So, combining Eqs. (19) and (20), we obtain:

$$
\frac{\partial U}{\partial T}+\Delta \frac{\partial U}{\partial \xi}-\pi \varepsilon U \frac{\partial U}{\partial \xi}-D \frac{\partial^{2} U}{\partial \xi^{2}}=\frac{M}{2} f(\xi-\pi) .
$$

Equation (21) was named the "inhomogeneous Burgers equation with a discrepancy" [13]. Its main properties were studied in [13-15].

\section{NON-STEADY-STATE SOLUTION FOR A SAWTOOTH-LIKE MOTION OF THE BOUNDARY}

Equation (21) can be solved exactly for the most interesting resonant case $(\Delta=0)$ for some special types of vibration of the boundary. These exact solutions can help in understanding the general physical properties of forced nonlinear vibrations of resonators; moreover, the corresponding motion of its wall can be realized in experiments.

Let the boundary execute a sawtooth-like periodic motion:

$$
f(\omega t)=\left(1-\frac{\omega t}{\pi}\right) \operatorname{sgn}(\omega t), \quad-\pi \leq \omega t \leq \pi .
$$

Seeking for the solution to Eq. (21) in the form

$$
U=-a(T) \frac{\xi}{\pi}, \quad f=-\frac{\xi}{\pi}, \quad-\pi \leq \xi \leq \pi,
$$

giving $\partial^{2} U / \partial \xi^{2}=0$, we obtain the ordinary differential equation

$$
\frac{\partial a}{\partial T}+\varepsilon a^{2}=\frac{M}{2} .
$$

Its solution

$$
a=\sqrt{\frac{M}{2 \varepsilon}} \tanh \left(\sqrt{\frac{\varepsilon M}{2}} T\right)
$$

describes the increase with the time $T$ in the amplitude of the auxiliary function $U$ (Eq. (23)) up to the limiting value $(M / 2 \varepsilon)^{1 / 2}$. 


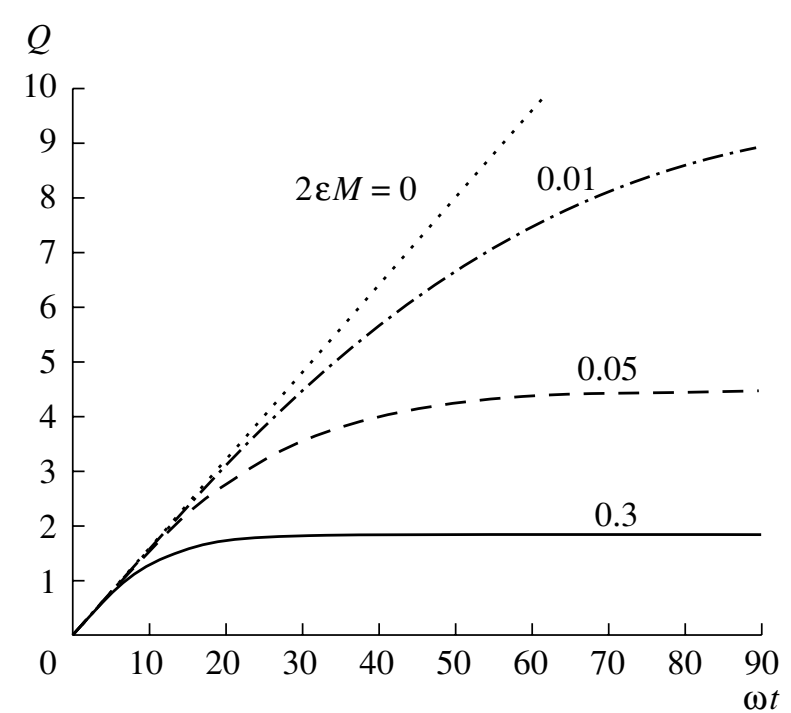

Fig. 2.

The form of the standing wave in the layer is determined by solution (11), where one can neglect small nonlinear terms, if the vibration within a time of order of one period is considered. Taking into account the periodicity of $F$, we write Eq. (11) as

$$
u=c U(\omega t-k x)-c U(\omega t+k x) .
$$

The temporal profiles of the standing wave given by Eqs. (23), (25), and (26) are shown in Fig. 1 for different cross-sections of the resonator: $x=L / 8, L / 4, L / 2$, $3 L / 4$, and $7 L / 8$. The form of forced nonlinear vibration of the particle velocity varies at the transition from one cross-section to another. In the middle of the layer, each period consists of alternating positive and negative rectangular regions, which have equal durations and "amplitudes".

At approaching the end $x=L$, the positive region becomes narrow, but its height increases up to the magnitude twice exceeding the magnitude in the middle point $x=L / 2$. The negative region decreases in magnitude, but expands in duration. The complete area (the integral of $u$ over the period) is equal to zero.

At approaching the other end, $x=0$, the narrowing and growth of the negative region take place. At both ends, $x=0$ and $L$, the motion disappears and $u=0$ in accordance with the boundary conditions (8) and (9). For the driving wall, this satisfaction of condition (8) should not be taken literally; it means that the amplitude of wall vibration is small in comparison with the characteristic magnitude of $u$ in the layer.

The mean volume density of acoustic energy

$$
\rho \overline{u^{2}}=\frac{\rho}{2 \pi} \int_{0}^{2 \pi} u^{2} d(\omega \tau)=\rho c^{2} \times 8 k x\left(1-\frac{k x}{\pi}\right) a^{2}(T)
$$

has a maximum at the center of the layer, $k x=\pi / 2$, and equals zero at both ends. The whole energy in the volume $V$ of the resonator, when calculated by integration of Eq. (27) over $x$, equals

$$
E=\frac{1}{3} \rho c^{2} V \frac{M}{\varepsilon} \tanh ^{2}\left(\sqrt{\frac{\varepsilon M}{2}} \frac{\omega t}{\pi}\right) .
$$

The ratio of "amplitudes" of the standing wave and the vibration of the boundary

$$
Q=\frac{a(T)}{M}=\frac{1}{\sqrt{2 \varepsilon M}} \tanh \left(\sqrt{2 \varepsilon M} \frac{\omega t}{2 \pi}\right)
$$

is shown in Fig. 2 for different values of $2 \varepsilon M$. At steady-state regime,

$$
Q=\left(2 \varepsilon \frac{A}{c}\right)^{-1 / 2} .
$$

If nonlinear effects were negligible, the $Q$-factor would not depend on the amplitude $A$ of excitation and would be equal to [12]

$$
Q=\frac{1}{2 D}=\frac{c^{3} \rho}{b \omega^{2} L}=\frac{c^{2} \rho}{\pi b \omega} .
$$

The linear $Q$-factor is determined by quite different physical quantities than the nonlinear one (Eq. (29)). In particular, it depends on the effective viscosity $b$ and on the frequency $\omega$.

For the amplitude $A=10 \mathrm{~cm} / \mathrm{s}$, the nonlinear evaluation is $Q \approx 37$. On the other hand, at typical frequencies about $4 \mathrm{kHz}$ in the air-filled resonator, the linear $Q$-factor is much higher: it is on the order of $10^{3}-10^{4}$. However, at high values of the linear $Q$-factor, the amplitude of the standing wave increases with time, and nonlinear absorption comes into play necessarily, leading to a considerable decrease in the $Q$-factor.

It must be noted here that some techniques are invented to suppress the nonlinear absorption $[1,2]$, but this paper is devoted to the common simplest resonators and more complicated systems are not considered here.

The excitation of the layer by a sawtooth-like motion (Eq. (22)) discussed above is similar to that at the harmonic excitation (see below). There exists, however, another form of sawtooth-like vibration providing a radically new regime of amplification of a standing wave. Let us consider the so-called "inverse saw" motion of the driving wall,

$$
U=a(T) \frac{\xi}{\pi}, \quad f=\frac{\xi}{\pi}, \quad-\pi \leq \xi \leq \pi,
$$

instead of the "common saw", Eq. (23). The ordinary equation for the amplitude $a(T)$

$$
\frac{\partial a}{\partial T}-\varepsilon a^{2}=\frac{M}{2}
$$


differs from Eq. (24) only by a sign, but it describes a radically different phenomenon:

$$
a=\sqrt{\frac{M}{2 \varepsilon}} \tan \left(\sqrt{\frac{\varepsilon M}{2}} T\right) .
$$

One can see that amplitude (30) increases indefinitely at

$$
\omega t \rightarrow \frac{\pi^{2}}{\sqrt{2 \varepsilon M}} \text {. }
$$

Such an "explosive instability" in the solution to the inhomogeneous equation (21) was discussed in [15]. This phenomenon can be explained as follows: the energy transmitted from the source to the smooth sections of the profile of the travelling wave $U$ does not have enough time to reach the leading shock front and to be dissipated at it. Therefore, the unlimited growth of amplitude (30) goes on, and only some other nonlinear phenomena (for example, the nonlinearity of the moving boundaries [2]) can be a possible mechanism of limitation. This regime must be investigated in more detail. Anyway, it seems promising for the pumping of high acoustic energy to a limited volume of the medium.

\section{STANDING WAVE EXCITED BY HARMONIC VIBRATION}

For the harmonic vibration of the wall, the righthand side of Eq. (21) takes the form $-(M / 2) \sin \xi$. For this case and some other cases, the linearization can help in solving the inhomogeneous Burgers equation (21). Using the transformation

$$
U=\frac{2 D}{\pi \varepsilon} \frac{\partial}{\partial \xi} \ln W
$$

one can reduce the nonlinear equation (21) to a linear one:

$$
\frac{\partial W}{\partial T}+\Delta \frac{\partial W}{\partial \xi}-D \frac{\partial^{2} W}{\partial \xi^{2}}=\frac{1}{2} q D \cos \xi W, \quad q=\frac{\pi \varepsilon M}{2 D^{2}} .
$$

By the substitution

$$
W=\exp \left(-\frac{1}{4} \lambda D T\right) y\left(z=\frac{\xi}{2}\right)
$$

where $\lambda$ is a constant, we derive an ordinary differential equation for the function $y(z)$ :

$$
\frac{\partial^{2} y}{\partial z^{2}}-2 \frac{\Delta}{D} \frac{\partial y}{\partial z}+(\lambda+2 q \cos 2 z) y=0 .
$$

At zero discrepancy, $\Delta=0$, Eq. (33) transforms to the canonical form of the differential equation for Mathieu's function. This is exactly the resonant case which must be studied in detail.
For zero initial condition $U(T=0, \xi)=0$, the solution to Eq. (32) can be written as a series of even Mathieu's functions [15]:

$$
W=\sum_{n=0}^{\infty} a_{2 n} \exp \left[-\frac{1}{4} \lambda_{2 n}(q) D T\right] c e_{2 n}\left(\frac{\xi}{2}, q\right),
$$

where

$$
a_{2 n}=\left[\int_{0}^{2 \pi} c e_{0}\left(\frac{\xi}{2}, q\right) d \xi\right] /\left[\int_{0}^{2 \pi} c e_{2 n}^{2}\left(\frac{\xi}{2}, q\right) d \xi\right] .
$$

The notations used here correspond to those from the book by Strutt [16] (see also [17]).

From Eqs. (31) and (34), the excitation of the travelling wave [15] $U(T, \xi)$ is found, and $U$ is here an auxiliary function for the construction of the standing wave profile during its approaching the steady-state condition. The stationary solution is the limit of Eq. (34) at $T \longrightarrow \infty$ [14]:

$$
U=\frac{2 D}{\pi \varepsilon} \frac{d}{d \xi} \ln c e_{0}\left(\frac{\xi}{2}, \frac{\pi \varepsilon M}{2 D^{2}}\right) .
$$

At $q \gg 1$, solution (35) takes the form [15]

$$
U=\sqrt{\frac{2 M}{\pi \varepsilon}}\left[\cos \frac{\xi}{2}-\frac{3 \exp (-2 \sqrt{q} \xi)}{1+2 \exp (-2 \sqrt{q} \xi)}\right], \quad 0 \leq \xi \leq \pi,
$$

and at $q \longrightarrow \infty$, it does not depend on the linear absorption (i.e., on $q$ ) at all:

$$
U=\sqrt{\frac{2 M}{\pi \varepsilon}} \cos \frac{\xi}{2} \operatorname{sgn} \xi, \quad-\pi \leq \xi \leq \pi .
$$

In Figs. $3 \mathrm{a}$ and $3 \mathrm{~b}$, the development of the periodic temporal profiles of the standing wave is shown for the harmonic vibration of the left-hand wall of the resonator, in the middle cross-section $(x=L / 2$, Fig. 3a) and near the right-hand end ( $x=7 L / 8$, Fig. $3 \mathrm{~b}$ ). The profiles are constructed after the calculation of series (34), with allowance for transformations (26) and (31), at the transient moments of slowly varying time: $D T=0.15,0.3$, 0.5 , and 0.9 . For the parameter $q$ (Eq. (32)), the value $q=20$ was taken.

The curves in Fig. 3, in contrast to the ones in Fig. 1, are smoother, because the formation of the shock front in the travelling wave $U$ at given moments $D T$ is in process. Even at $D T \longrightarrow \infty$, the shock front for $q=20$ will have a finite width.

At $D T \longrightarrow \infty$, the standing wave reaches its stationary condition. The steady-state profiles are smoothed at finite magnitudes of the parameter $q$, but discontinuities appear at $q \longrightarrow \infty$.

The steady-state profiles are shown in Fig. 4 for an extremely high harmonic excitation corresponding formally to $q \longrightarrow \infty$. The sum (26) of two travelling waves 

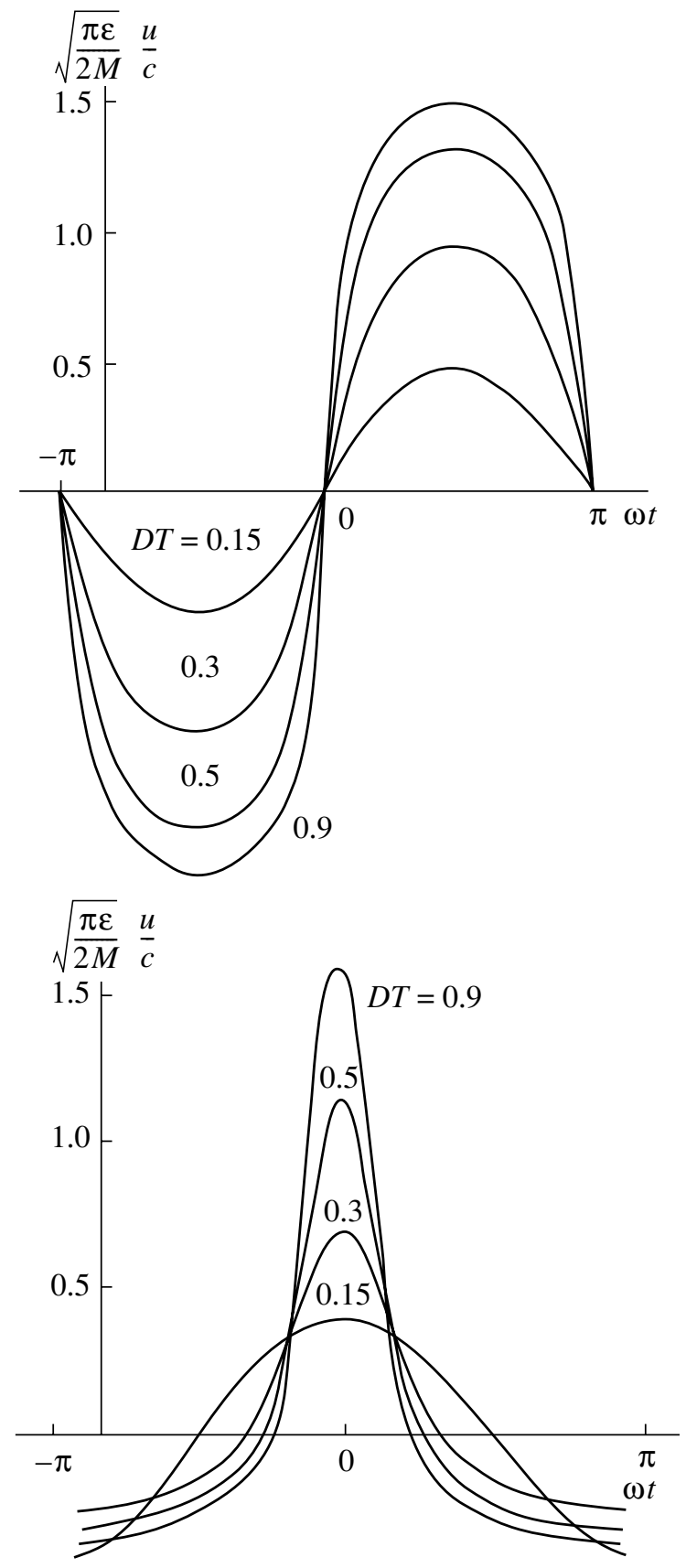

Fig. 3.

described by Eq. (36) is used. The standing wave in Fig. 4 has the shape similar to that in Fig. 1 for sawtooth-like excitation. Only the tops of the positive and negative pulses here are not flat; their form of an arc is described by trigonometric functions. In Fig. 1, the maximal peak of $u / c$ tends (at $T \longrightarrow \infty$ ) to $\sqrt{2 M / \varepsilon}$, and in Fig. 4 it equals to $2 \sqrt{2 M / \pi \varepsilon}$.

The calculation of the acoustic energy accumulated in the resonator is more complicated here than for sawtooth-like excitation (see above). The steady-state solu- tion (35) is described by the ordinary differential equation

$$
D \frac{d^{2} U}{d \xi^{2}}+\pi \varepsilon U \frac{d U}{d \xi}=\frac{M}{2} \sin \xi,
$$

which follows from Eq. (21) at $T \longrightarrow \infty, \Delta=0$ and $f(\xi)=-\sin \xi$. Integrating Eq. (37), we obtain

$$
D \frac{d U}{d \xi}+\frac{\pi \varepsilon}{2}\left(U^{2}-C\right)=-\frac{M}{2} \cos \xi \text {. }
$$

Since the mean value is $\bar{U}=0$, it follows from Eq. (38) that the constant $\mathrm{C}$ is equal to

$$
C=\overline{U^{2}}=\frac{1}{2 \pi} \int_{0}^{2 \pi} U^{2}(\xi) d \xi .
$$

Thus, the constant $C$ is proportional to the density of acoustic energy. Using transformation (31), we reduce the nonlinear equation (38) to a linear one for Mathieu functions:

$$
\frac{d^{2} W}{d z^{2}}+\left[-\left(\frac{\pi \varepsilon}{D}\right)^{2} C+\frac{\pi \varepsilon M}{D^{2}} \cos 2 z\right]=0,
$$

where $z=\xi / 2$. Comparing Eqs. (39) and (33), we conclude that the energy is proportional to the eigenvalue $\lambda_{0}$ of the Mathieu function $c e_{0}[14]$ :

$$
\overline{U^{2}}=-\left(\frac{D}{\pi \varepsilon}\right)^{2} \lambda_{0}\left(q=\frac{\pi \varepsilon M}{2 D^{2}}\right) .
$$

The total energy of the resonator, in accordance with formulas (26) and (27), equals to $E=\rho c^{2} V \times 2 \overline{U^{2}}$.

At a weak excitation $\lambda_{0} \approx-q^{2} / 2$ [16], a well-known linear result appears:

$$
E \approx\left(\frac{M}{2 D}\right)^{2} \rho c^{2} V .
$$

Using another asymptotics for the eigenvalue $\lambda_{0}$ at $q \gg 1$ [17], for strong boundary vibration we derive

$$
E=\left[\frac{2 M}{\pi \varepsilon}-\frac{2 D}{(\pi \varepsilon)^{2}} \sqrt{2 \pi \varepsilon M}+\frac{1}{2} \frac{D^{2}}{(\pi \varepsilon)^{2}}+\ldots\right] \rho c^{2} V .
$$

When $q \longrightarrow \infty$, the factor $2 / \pi$ in the first leading term in brackets in Eq. (40) differs from the result given by Eq. (28), where at $T \longrightarrow \infty$ the corresponding factor is equal to $1 / 3$.

Thus, the comparison of the results for sawtoothlike and harmonic excitations described above shows their qualitative similarity.

Using solution (34) supplemented by transformations (26) and (31), one can calculate numerically all other characteristics of the standing waves in the layer for both the transient regime and the steady-state one. 


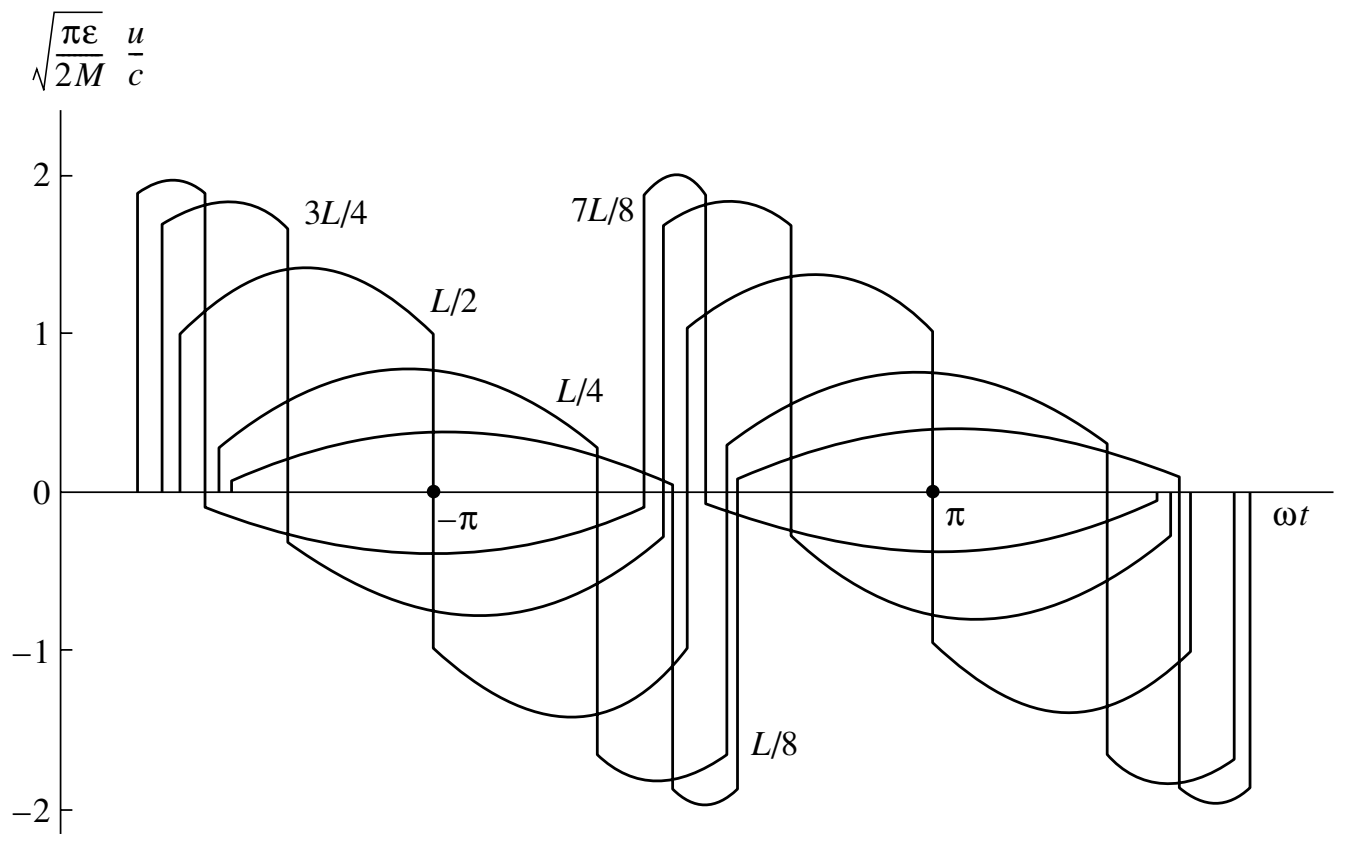

Fig. 4.

The arbitrary relationship between nonlinearity and competitive dissipation processes can be considered. In this paper, however, we have restricted our consideration to the description of the new approach and to the most general analytical results. More particular numerical studies can be performed as a continuation of this work.

\section{CONCLUSIONS}

A new analytical approach to the theory of nonsteady-state forced vibration in a layer of a nonlinear dissipative medium is developed on the basis of the simplified evolution equation for the auxiliary function describing two nonlinear waves propagating in opposite directions. This approach is explained and justified for the periodic vibration of a wall. A new equation is derived for standing waves, which is known in the nonlinear wave theory as the "inhomogeneous Burgers equation with a discrepancy". Three types of the wall motion are considered: (i) the vibration of sawtoothlike form, (ii) the vibration of the form of an "inverse saw", and (iii) the harmonic vibration. For the "inverse saw" motion, the "explosive instability" is progressing in the resonator, leading to an infinite increase in the standing wave amplitude within a finite time. For the other two regimes, i.e., the sawtooth-like and the harmonic regimes, the transient and steady-state vibrations are studied. The temporal profiles are constructed for different cross-sections of the layer. The nonlinear $Q$-factor and the total energy in the resonator are calculated. For the harmonic motion, the main characteristics can be expressed through Mathieu functions and their eigenvalues.

\section{ACKNOWLEDGMENT}

This work was supported by the Royal Swedish Academy of Sciences, the Russian Foundation for Basic Research, and the INTAS (project no. 97-11134), as well as by a grant under the program "Universities of Russia."

\section{REFERENCES}

1. C. C. Lawrenson, B. Lipkens, T. S. Lucas, et al., J. Acoust. Soc. Am. 104, 623 (1998).

2. O. V. Rudenko, Akust. Zh. 45, 397 (1999) [Acoust. Phys. 45, 351 (1999)].

3. W. Chester, J. Fluid Mech. 18, 44 (1964).

4. S. Temkin, J. Acoust. Soc. Am. 45, 224 (1969).

5. A. B. Coppens and A. A. Atchley, in Encyclopedia of Acoustics (Wiley, New York, 1997), pp. 237-246.

6. M. A. Ilhamov, R. G. Zaripov, R. G. Galiullin, and V. B. Repin, Appl. Mech. Rev. 49 (3), 137 (1996).

7. V. V. Kaner, O. V. Rudenko, and R. V. Khokhlov, Akust. Zh. 23, 756 (1977) [Sov. Phys. Acoust. 23, 432 (1977)].

8. V. V. Kaner and O. V. Rudenko, Vestn. Mosk. Univ., Fiz., Astron., No. 4, 78 (1978).

9. M. B. Vinogradova, O. V. Rudenko, and A. P. Sukhorukov, The Theory of Waves, 2nd ed. (Nauka, Moscow, 1990). 
10. O. V. Rudenko and S. I. Soluyan, Theoretical Foundations of Nonlinear Acoustics (Nauka, Moscow, 1975; Consultants Bureau, New York, 1977).

11. O. V. Rudenko and A. V. Shanin, Abstracts of Papers, ISNA-15, Goettingen, 1999 (Academic, New York) (to be published).

12. O. V. Rudenko and A. V. Shanin, Akust. Zh. 46, 392 (2000) [Acoust. Phys. 46, 334 (2000)].

13. A. A. Karabutov and O. V. Rudenko, Akust. Zh. 25, 536 (1979) [Sov. Phys. Acoust. 25, 306 (1980)].
14. O. V. Rudenko, Pis'ma Zh. Éksp. Teor. Fiz. 20, 445 (1974) [JETP Lett. 20, 203 (1974)].

15. A. A. Karabutov, E. A. Lapshin, and O. V. Rudenko, Zh. Éksp. Teor. Fiz. 71, 111 (1976) [Sov. Phys. JETP 44, 58 (1976)].

16. M. J. O. Strutt, Lame, Mathieu and Related Functions in Physics and Technology (Springer, Berlin, 1932; ONTI, Moscow, 1935; Edwards Brothers, New York, 1944).

17. Handbook of Mathematical Functions, Ed. by M. Abramowitz and I. A. Stegun (Dover, New York, 1970; Nauka, Moscow, 1979). 